\title{
PSICOTERAPIA ON LINE II Revisión a partir de la experiencia y la investigación
}

\author{
Josep Mercadal y Victor Cabré \\ Fundació Vidal i Barraquer, Barcelona
}

\begin{abstract}
El año 2016 escribimos un trabajo ${ }^{1}$, sobre el mismo tema que tratamos aquí, con el que pretendíamos animar a reflexionar y comprender las variables que diferenciaban la terapia psicológica on line y la presencial. Por aquel entonces, nuestra experiencia con los tratamientos online representaba una porción muy reducida de nuestra actividad asistencial. En 2020 la pandemia mundial provocada por el COVID 19 invirtió, radicalmente, estos porcentajes y obligó a "convertir" la mayor parte de la actividad psicoterapéutica a la metodología on line.

En este nuevo trabajo, hemos hecho una revisión de las investigaciones publicadas en estos últimos años, además de seguir reflexionando sobre este recurso técnico que con la ayuda de la pandemia se ha instalado, irremediablemente, en nuestras consultas. Aspectos como el encuadre, la formación e investigación al respecto, son analizados en este texto.
\end{abstract}

Palabras clave: Intervención on line, formación, investigación, encuadre.

In 2016 we wrote a paper, on the same topic that we deal with here, with which we intended to encourage reflection and understanding of the variables that differentiate online and face-to-face psychological therapy. At that time, our experience with online treatments represented a very small portion of our healthcare activity. In 2020, the global pandemic caused by COVID 19 radically inverted these percentages and forced to "convert" most of the psychotherapeutic activity to online methodology.

In this new work, we have reviewed the research published in recent years, in addition to continuing to reflect on this technical resource that, with the help of the pandemic, has been irretrievably installed in our consultations. Aspects such as setting, training, research, etc. are analysed in this text.

Key Words: Online intervention, training, research, setting

English Title: ON LINE PSYCHOTHERAPY (II) Review based on experience and research

\section{Cita bibliográfica / Reference citation:}

Mercadal, J. y Cabré, V. (2021). Psicoterapia On line II. Revisión a partir de la experiencia y la investigación. Clínica e Investigación Relacional, 15 (2): 359-371. [ISSN 1988-2939] [Recuperado de www.ceir.info ] DOI: 10.21110/19882939.2021.150204

\footnotetext{
${ }^{1}$ Cabré, V. y Mercadal, J. (2016). Psicoterapia On-line. Escenario Virtual para una relación real en el espacio intermediario. Clínica e Investigación Relacional, 10 (2): 439-452. [ISSN 1988-2939] [Recuperado de www.ceir.info] DOI: 10.21110/19882939.2016.100206
} 


\section{Introducción}

Para ayudar a otro ser humano que sufre, las mejores condiciones pasan por estar cerca de él, en un mismo espacio y durante un tiempo en el que ambos puedan sentir suficiente seguridad para confiar plenamente en ese encuentro con el otro. Esas condiciones son las que facilitarán que se puedan desarrollar procesos nucleares de la relación asistencial como la Alianza Terapéutica y permitirán la adecuada comprensión de distintas variables intervinientes como la transferencia y la contratransferencia. Somos conscientes de que estas condiciones del encuadre no siempre son posibles y entonces, nuestra responsabilidad como profesionales, pasa por observar, comprender y valorar qué nuevas variables inciden en la relación para que el resultado siga siendo el mejor posible.

El año 2016 escribimos un trabajo, sobre el mismo tema que tratamos aquí, con el que pretendíamos animar a reflexionar y comprender las variables que diferenciaban la terapia psicológica on line y la presencial. Hasta ese momento, nuestra experiencia con los tratamientos en línea representaba una porción comparativamente reducida de nuestra actividad asistencial y se centraba, principalmente, en la atención a personas desplazadas por motivos laborales o académicos $y$, en menor proporción, por motivos familiares, económicos o políticos. En estos casos procurábamos tener un conocimiento presencial previo del paciente (Cabré y Mercadal, 2016) y si esto no era posible, valorábamos con él la opción de derivarlo a otro profesional cercano a su nuevo lugar de residencia. Otra parte importante de la actividad con esta metodología en línea la ocupaban entrevistas de orientación o derivación y sesiones de seguimiento una vez finalizada la psicoterapia. Por supuesto que, a la muestra de ese momento, deberíamos añadir un reducido grupo de personas que, ya sea por las características especiales de su momento vital o de su cuadro psicopatológico, se hacía inviable cualquier otro tipo de intervención. Probablemente esta era la situación compartida por la mayoría de los profesionales de la psicoterapia hasta entonces, a excepción, claro está, de los servicios específicos de teleasistencia, telemedicina, etc, que trabajan con esta metodología desde hace tiempo.

En 2020 la pandemia mundial provocada por el COVID 19 invirtió, radicalmente, estos porcentajes y obligó a "convertir" la mayor parte de la actividad psicoterapéutica a la metodología on line. En muy poco tiempo, los terapeutas (y los pacientes) tuvimos que adaptarnos a unas condiciones que nos "obligaban" a modificar algunas variables (especialmente del encuadre) sin haberlo previsto previamente y sin ser totalmente conscientes de qué otros cambios, además de los tecnológicos, deberíamos afrontar.

Desde entonces ha habido períodos, más o menos prolongados, en los que hemos ido recuperando la presencialidad, simultaneándola con la actividad en línea. Buena parte de 
los casos que se iniciaron presencialmente están regresando al encuadre inicial, pero en otros muchos, ya no se recuperará la presencialidad y es posible que cada vez más nos encontremos con pacientes que deseen empezar, desarrollar y acabar un tratamiento en línea. Sinceramente desconocemos de qué forma, esta crisis, puede redistribuir el número de intervenciones, presenciales o en línea, que realizaremos en un futuro, una vez superada ésta, pero de lo que sí estamos convencidos es de la necesidad de comprender y evaluar las "nuevas" variables introducidas por ella, para que podamos diferenciar cuando adoptamos una metodología "por necesidad" o por ser una "indicación preferente" a ojos del profesional y de la comunidad científica.

En nuestro artículo del 2016 apuntábamos que la psicoterapia on line no constituía una nueva modalidad de intervención terapéutica, sino la utilización de un recurso técnico que puede ser adaptado para cualquiera de las modalidades descritas y manualizadas hasta el momento (Cabré y Mercadal, 2016), pero ya postulábamos dos de los retos que se abrían ante nosotros y que deberíamos encarar como profesionales: la formación y la investigación sobre este nuevo recurso.

\section{Eficacia de las Intervenciones}

Manfrida, Albertini y Eisenberg (2018) advierten de que el uso de todos estos recursos digitales no está exento de consecuencias en la relación paciente-terapeuta y que los terapeutas deben emplear, con gran cuidado y conocimiento, estos nuevos dispositivos comunicativos; más aun desconociendo los riesgos que podrían comportar para el paciente y la relación terapéutica. La prudencia de estos autores se nos antoja, también a nosotros, repleta de sentido común, pues la literatura existente hasta la fecha sigue siendo escasa y no hará falta esperar mucho más para empezar a obtener los primeros datos y, con ellos, las primeras respuestas.

Autores como Zwerenz, Becker, Knickenberg, Siepmann, Hagen, y Beutel (2017), Ilevaron a cabo un estudio en el que tenían como objetivo evaluar si los tratamientos combinados (presencialmente y vía on line) daban mejores resultados que aquellos únicamente presenciales, con pacientes diagnosticados de depresión y/o ansiedad. Para hacerlo, contaron con una muestra de 229 pacientes que fue dividida en dos grupos: aquellos pacientes que recibían tratamiento combinado y los pacientes que recibían solamente tratamiento presencial. Los resultados apuntaban a que los pacientes depresivos, que habían recibido el tratamiento combinado, habían mejorado significativamente respecto a los que habían recibido tratamiento convencional. Los mismos resultados se obtuvieron con los pacientes ansiosos, concluyendo así, que ésta es la primera evidencia de que el 
tratamiento on line funciona y animando a seguir investigando sobre los factores que contribuyen a su eficacia. Sin embargo, un estudio realizado por Rollman, Herbeck y Abebe (2018), en el que comparaban la aplicación de tratamientos vía online y presencial, con una muestra de 704 pacientes que también sufrían de ansiedad y/o depresión, concluyeron que la terapia on line no proporcionaba ningún beneficio adicional a la terapia presencial. Vemos, por tanto, que la poca literatura existente hasta el momento todavía es poco concluyente. En este punto, no podemos evitar recordar el debate a propósito de otro tradicional "tratamiento combinado": el que está integrado por un tratamiento psicofarmacológico y otro psicoterapéutico. En este caso la investigación ha decantado la balanza de la eficacia hacia los mejores resultados del tratamiento combinado en la mayoría de los trastornos graves (psicosis) así como en buena parte de trastornos, con menor gravedad objetiva (depresión, ansiedad), pero en los que se requería una intervención farmacológica. El análisis en detalle de este último grupo apuntaba en la dirección de que el paciente se sentía mejor atendido, contenido y comprendido, cuando dos tratamientos (y tal vez dos profesionales) se ocupaban de su malestar colaborando conjuntamente. Debemos ser cautos y esperar a disponer de más información al respecto, pero no sería descartable que factores como la inmediatez (propia del contacto en línea), pueda jugar un papel importante en la mejoría, sobre todo en una evaluación a corto plazo.

\section{Necesidades de Formación}

Una de las características comunes a todas las nuevas tecnologías consiste en asegurar su facilidad de uso. Como si de un eslogan comercial se tratara, el éxito de su implementación pasa por asegurar que, en muy poco tiempo, cualquier usuario puede incorporarlas fácilmente a su actividad profesional. Tal vez haya algo de cierto en ello ya que, de hecho, las posibilidades y herramientas tecnológicas al servicio de la psicoterapia en línea se han multiplicado rápidamente. Aun así, creemos que el riesgo proviene de pensar que se trata de hacer exactamente lo mismo, solo que a través de una plataforma. Incluso han aparecido grupos de profesionales que, aprovechando estas nuevas posibilidades, han imaginado que se facilitan gestiones y se abre la oportunidad de reducir costes concentrando toda su actividad exclusivamente en la metodología en línea. Proponemos que el terapeuta adquiera un conocimiento más profundo de la herramienta que se propone utilizar: además de las cuestiones tecnológicas, conviene que conozca las implicaciones éticas relacionadas con la intimidad, la protección de datos, el consentimiento informado etc. También es necesario complementar sus conocimientos sobre las teorías de la comunicación en las que están implicados elementos verbales y no 
verbales, cenestésicos y corporales, analógicos y digitales, etc. En definitiva, aspectos que han estado siempre presentes en la relación asistencial pero que, ahora, aparecen mediatizados por un nuevo entorno que los acentúa, atenúa, transforma, parcializa, etc.

Una de las experiencias compartidas, a menudo, entre profesionales hace referencia al mayor cansancio experimentado con la actividad en línea. Es muy posible que intervengan factores como la mayor tensión provocada por la novedad, por el desconocimiento, por la existencia de un nuevo elemento que requiere de nuestra atención. Desde nuestra perspectiva pensamos que, además, los mismos elementos básicos de la relación asistencial (alianza terapéutica, transferencia y contratransferencia, etc.) se manifiestan ahora en una suerte de transformación que a veces contrae, concentra o diluye; como si el medio reclamara un incremento de atención y de tensión para compensar la "novedad" pero, sobre todo, la mayor "distancia". Aun así, también es cierto que nuestra experiencia nos lleva a afirmar que cuando este plus de esfuerzo (tanto por parte del terapeuta como del paciente) resulta efectivo en este intento "compensatorio", pueden producirse los momentos "ahora" (en los que la "sorpresa" facilita el insight) de la misma forma -o mejor dicho, con la misma intensidad y eficacia para promover cambios y transformaciones- que en cualquier otra modalidad de intervención. En psicoterapia, además, siempre que hacemos referencia a la formación de los profesionales, debemos incorporar la supervisión. Probablemente, a partir de ahora, en el estudio y análisis de un caso incorporaremos la reflexión de si es conveniente o no el uso de la metodología en línea, en qué circunstancias y bajo qué condiciones, al tiempo que la supervisión adquiera un papel preponderante.

En esta línea, el Consejo General de la Psicología de España, ha elaborado una Guía de Buenas Prácticas de la Tele-psicología, tomando como base las directrices de la American Psychologist Association (APA, 2013) en donde se relacionan algunos aspectos específicos que deberían tenerse en cuenta:

1. En la evaluación del paciente incluir:

- Distancia geográfica al centro médico de urgencias más cercano

- Eficacia del sistema de apoyo del paciente

- Estatus médico actual

- Nivel general de competencia tecnológica

2. En relación al Consentimiento Informado:

- Incluir información específica relacionada con la naturaleza de los servicios de videoconferencia 


\section{Otros aspectos técnicos del encuadre:}

- Verificación identidad del paciente y del profesional (en el primer encuentro entre ambos)

- Verificación del lugar del paciente, en relación a la posibilidad de tener que poner en marcha el protocolo de urgencias (intentando que el lugar sea estable en futuros encuentros)

- Necesidad de contar con un plan de apoyo en el caso de que se produzca un fallo técnico que interrumpa la sesión (a través de una llamada telefónica o correo que permitan "cerrar" la sesión)

En algunos países como Brasil, sólo se permite la realización de intervenciones psicológicas on line si es para investigar sobre su eficacia (Pieta y Gomes, 2014), argumentando que este nuevo recurso técnico puede tener limitaciones o problemas legales y éticos relacionados con su práctica. En este mismo sentido, Stoll y Trachsek (2019), afirman que los argumentos éticos más importantes debatidos en la literatura se analizan revisando, por un lado, los argumentos a favor de la psicoterapia on line, como un mayor acceso a la atención, un elemento que puede complementar el tratamiento, el anonimato y la rentabilidad y, por otro lado, argumentos en contra de la psicoterapia on line, como el riesgo de deterioro del tratamiento, problemas de confidencialidad, problemas con el consentimiento informado, identificación del paciente y situaciones de emergencia, problemas legales y habilidades adicionales necesarias (tecnológicas); aunque ambos estudios concluyen que las cuestiones jurídicas y éticas pueden ser parcialmente resueltas con claras directrices de las organizaciones profesionales y formación de los terapeutas en la modalidad on line. Una de las advertencias contenidas en la guía citada anteriormente (APA, 2013) es: "Cada país o estado puede tener su propia jurisdicción o legislación específica a la hora de proporcionar servicios a través de tecnologías de la comunicación. La legislación del lugar en que se encuentra el paciente es relevante, ya que puede exigirse que la titulación del profesional de la psicología esté acreditada en ese territorio o país".

\section{Generando Opinión}

Drath y Necki (2018), llevaron a cabo una investigación en la que estudiaban las actitudes de los psicoterapeutas polacos hacia el fenómeno de la psicoterapia on line. Administraron cuestionarios a un total de 193 terapeutas y los resultados reflejaron que casi un tercio (32\%) de los psicoterapeutas han llevado a cabo o están ofreciendo psicoterapia en línea. Además, la gran mayoría de ellos (77.4\%), cree que la psicoterapia on line debe usarse solo en casos excepcionales y el $10.3 \%$ de los encuestados declaró que la psicoterapia on line no 
debería realizarse en absoluto. Por lo tanto, estos resultados atestiguan el proceso continuo de reflexión profesional sobre la nueva fórmula de contacto con el paciente. Además de que los terapeutas evalúan claramente (positiva o negativamente) el fenómeno estudiado, hay un gran grupo de profesionales que expresan sus dudas sobre esta forma de terapia y exigen su uso solo en situaciones excepcionales claramente especificadas.

Por su parte, en otro trabajo llevado a cabo por investigadores alemanes (Schulze, Reuter, Kuchler, Reinke, Heinkelmann, Stöckigt, Siemoneit y Tonn, 2019), que reconocen que el estudio de esta técnica en su país se encuentra en sus primeras fases, quisieron evaluar las diferentes actitudes hacia las intervenciones on line entre los profesionales de la salud mental y los no profesionales. Para hacerlo, contaron con una muestra de 97 profesionales y 1.353 no profesionales. Los resultados mostraban que los no profesionales calificaban el uso de las intervenciones on line de manera más crítica que los profesionales, lo que evidencia el dudoso criterio que se sigue, a veces, por parte del profesional, a la hora de decidir llevar a cabo una intervención on line.

Por el momento, las organizaciones de profesionales se muestran prudentes, al mismo tiempo que ofrecen todo su esfuerzo y colaboración para facilitar las mejores condiciones a profesionales y pacientes. Asociaciones y colegios profesionales están publicando guías específicas para el trabajo en línea que recogen un buen número de recomendaciones. En el ámbito de la medicina, por ejemplo, se recuerda: "Debe tenerse en cuenta lo señalado en el artículo 26.3 del vigente Código de Ética y Deontología Médica, que recuerda que el ejercicio clínico de la medicina mediante consultas exclusivamente por carta, teléfono, radio, prensa o internet, es contrario a las normas deontológicas, lo que podría conllevar en determinados supuestos al ejercicio del conveniente poder disciplinario, al entenderse que la actuación correcta, desde un punto de vista médico, implica de forma ineludible el contacto personal y directo entre el médico y el paciente. Ello no significa que una atención por medios electrónicos (online) esté prohibida, pero en estos momentos, resulta obvia la necesidad de considerarla un "complemento" con respecto de un adecuado ejercicio profesional que implica el contacto en consulta física".

\section{Encuadre Externo / Encuadre Interno}

A menudo, en el debate entre modalidad en línea o presencial, se encuentra implícita la pregunta de si, el vínculo, será el mismo: más o menos sólido, más o menos estable o más o menos profundo. No hemos encontrado ninguna razón para pensar que el mejor encuadre posible para la relación asistencial sea la videoconferencia (de la misma forma que, probablemente, tampoco lo sea el hogar familiar de un paciente o la sala compartida 
de una institución). Todas ellas, y tantas otras, son situaciones no solamente posibles sino habituales y en un gran número de casos hasta pueden ser las mejores bajo determinadas condiciones. Todas ellas, además, pueden aproximarse, en mayor o menor medida, a esas condiciones de seguridad y confianza tan necesarias para favorecer el vínculo, obteniendo los mismos objetivos y resultados. Pero, precisamente porque las condiciones son distintas, no deberíamos situarnos frente a ellas de la misma forma: como terapeutas nos "posicionamos" de forma distinta si sabemos que disponemos de unos pocos minutos, si el entorno es ruidoso o poco privado, si compartimos el mismo espacio con el paciente o si nos asomamos a la pantalla.

Si, como ya se ha propuesto en otros trabajos (Cabré y Mercadal, 2016; Vaimberg, 2014) la pantalla puede ser comprendida en términos de espacio transicional, la calidad de la relación entre terapeuta y paciente parece que quedaría preservada, a pesar de tratarse de una distancia mayor, como ya se ha dicho. Creemos que todavía nos queda mucho por entender a propósito de esta paradoja: la metodología en línea está pensada para acercar, pero también nos aleja. Ribé (2021), refiriéndose a la empatía se pregunta, no sin cierta ironía, si las neuronas espejo responden igual en un medio virtual que en uno presencial. Comprender las variables que incorporan cada una de estas modalidades del encuadre, facilitará nuestra forma de situarnos en cada una de ellas.

De momento, el Colegio Oficial de Psicólogos de Madrid (Torre y Pardo, 2018) propone: "La asistencia a distancia se recomienda implementar cuando, por el contexto y características de la persona, sea la única o la mejor manera de proporcionar dicha asistencia" y añade:

"El psicólogo/a tiene la máxima responsabilidad en la toma de decisiones y ajuste individual para mantener la calidad, eficacia y seguridad de la intervención. Por este motivo, no hay absolutas contraindicaciones sobre las personas a las que atender, pero sí se deben atender las siguientes recomendaciones:

Se aconseja la intervención telepsicológica cuando es:

- La única opción para la persona de recibir ayuda psicológica y no existe opción presencial posible o recomendada.

- Está justificado y es equivalente o mejora al tratamiento presencial.

- Las condiciones clínicas (tipo de problema, sin riesgo o crisis, gravedad leve o media, no requiere estrategias presenciales específicas) lo permiten.

- Se cumplen los criterios básicos (mayor de 16 años, buen manejo tecnológico, espacio y privacidad adecuada...) 
- Se adapta a las condiciones de trabajo terapéutico (tareas y ejercicios, sin impedimentos en la comunicación ni incongruencias, sin empeoramiento o riesgo, se producen avances clínicos).

Se desaconseja la intervención telepsicológica cuando se incumplen las anteriores y en las siguientes condiciones clínicas:

- Muy bajo estado de ánimo.

- Ansiedad excesiva, muy pocas estrategias de autocontrol emocional, crisis frecuentes.

- Adicciones, problemas de alimentación o de control de impulsos graves.

- Ideación suicida, riesgo autolítico o problemas de violencia, abuso o acoso.

- Conflictos familiares/pareja importantes.

- Pérdida de realidad, alucinaciones, delirios, etc.

- Inhabilidad social grave/agorafobia - requiere estrategias específicas.

Por nuestra parte, hemos observado que se han multiplicado las circunstancias que pueden motivar el cambio de metodología asistencial. Si antes de la pandemia contemplábamos que se podía pasar de lo presencial a lo on line por causas relativas al paciente que se desconocían al iniciar la intervención y, lo mismo, pero por causas relativas al terapeuta, ahora vemos que han aparecido y casi se han "normalizado" muchas otras circunstancias que incrementan esta casuística y la hacen más compleja:

- Pacientes que empiezan un tratamiento presencial sabiendo que, en algún momento, va a tener que continuar vía online.

- Pacientes que empiezan online sabiendo que, a partir de un determinado momento, podrán continuar presencialmente.

- Pacientes que empiezan online y desean mantener esta modalidad durante todo el tratamiento.

- Pacientes que empiezan presencialmente, se adopta la modalidad online por alguna razón temporal y ya no desean regresar a la presencialidad aun siendo ésta posible.

- Pacientes que deciden interrumpir el tratamiento ante la imposibilidad de seguir presencialmente.

- Pacientes que abandonan el tratamiento si se les "niega" (después de valorar posibles contraindicaciones) la posibilidad de seguir online. 
Hemos detectado que muchos pacientes, por un tema logístico o simplemente de comodidad, prefieren empezar y terminar la psicoterapia en línea. Durante la pandemia, cuando no había opción de elegir debido al confinamiento, era incluso positivo que el paciente quisiera hacerlo así, ya que, de lo contrario, no existía alternativa. Una vez se han relajado o eliminado las medidas de distanciamiento social, siguen siendo muchos los pacientes que piden realizar toda la intervención en línea. En estos casos, debemos advertirles de que la mejor indicación, si existe la posibilidad, es realizar al menos, las primeras sesiones de forma presencial, más aún si se prevé que el tratamiento va a continuar de forma telemática. Esto responde a la necesidad de poder dar un sentido más amplio y personal a la representación del otro: si antes hemos podido conocer su manera de relacionarse con nosotros de forma presencial, será más fácil que podamos interpretar sus gestos, expresiones, silencios, pausas, etc. a través de la pantalla.

También debemos hacer referencia a aquellos pacientes que no han aceptado la propuesta de relación en línea como forma predeterminada. Hemos visto a pacientes que se sienten más seguros hablando desde su casa a través de la pantalla, ya que ésta les da una seguridad que la presencialidad les niega; la pantalla actúa como protección a los miedos y ansiedades. Sin embargo, también hemos visto a pacientes cohibidos frente al hecho de hablar abiertamente, por miedo a que algunas de las persones que conviven con él le puedan escuchar. En estos pacientes, el confinamiento producido por la pandemia les ha afectado doblemente: ha generado o evidenciado conflictos relacionales con la pareja, la familia o personas con las que convive, por un lado y, por otro, la imposibilidad de realizar la consulta presencialmente y tener que hacerlo de forma on line, ha incrementado aún más su malestar por el miedo a que pudieran escucharle hablando con su terapeuta. Esta circunstancia puede estar presente, también, en el caso del terapeuta que se conecta desde su propio hogar, en vez de un despacho, y verse frenado de alguna forma al estar en un espacio compartido con más personas. Por lo tanto, el hecho de realizar una intervención psicológica con alguno de los participantes conectado desde su propia vivienda creemos que condiciona en exceso la intimidad de la sesión y, con ella, la evolución del tratamiento.

En nuestro anterior trabajo (Cabré y Mercadal, 2016) reflexionábamos sobre distintos elementos característicos de la comunicación en línea. Por un lado, en cuanto al uso de la voz y de los silencios, habíamos detectado que en las intervenciones en línea se pronunciaban frases más cortas y sintéticas, potenciando así lo racional en detrimento de lo emocional o relacional y con la pandemia hemos constatado que efectivamente esto es así, sobre todo en primeras sesiones, visitas de asesoramiento o exploración, mientras que, en las psicoterapias, ya sean empezadas presencialmente o en línea, se toleran mejor los silencios. Por otra parte, en cuanto al aspecto físico y la imagen, hemos observado hasta 
cuatro subtipos de paciente en función de cómo se colocan ante la cámara para realizar las sesiones: 1) los que se acercan tanto a la cámara que no dejan espacio, en la pantalla, para ver nada más, 2) los que sitúan la cámara lejos de ellos y donde se aprecia mejor su habitación o el espacio en el que están que ellos mismos, 3) los que, por distintos motivos, en cada sesión te enseñan una parte distinta de la casa (y no nos referimos a niños, a los cuales les gusta hacerlo, sino a adultos que por otras razones deben desplazarse), 4) los que ponen un fondo de pantalla impidiendo la observación de cualquier otra cosa detrás suyo ( $y$, quien sabe, si mostrándonos un lugar distinto en el que preferirían estar). Es evidente que el hogar puede estar impregnado de símbolos y otros elementos que, a menudo, incorporan motivos de distracción e interferencia en las sesiones en línea: la presencia de otras persones en el mismo espacio, de mascotas que reclaman la atención, Ilamadas a la puerta, electrodomésticos en funcionamiento, etc.

Finalmente quisiéramos llamar la atención sobre otro aspecto, que puede parecer menor, pero que tiene que ver con el tempo necesario para procesar los contenidos y las adquisiciones en un tratamiento. Al realizar las sesiones en línea desaparece el trayecto, de ida y vuelta, hasta la consulta y todos los que hemos realizado nuestro trabajo personal con un terapeuta, sabemos de la importancia que tiene el trayecto hasta su despacho pero, sobre todo, el trayecto de vuelta desde la consulta. Es importante y terapéutico el tiempo (aunque se trate de pocos minutos) en que estamos solos con nosotros mismos, después de la sesión. Es en ese espacio dónde se consolidan algunas de las ideas aparecidas en sesión o incluso es un momento de relajación en el que aparece el insight. En definitiva, es un momento que, en el caso de las sesiones en línea, no existe y que es una pérdida importante para el paciente, a no ser que, siendo consciente de ello, pueda reservarse para sí mismo unos minutos pre y post sesión.

\section{Coda}

Un trabajo como este, sobre las intervenciones en línea, corre el riesgo de quedar desfasado incluso antes de ser publicado. Esto es posible e incluso deseable, si se debe a la aparición de nuevos trabajos de investigación y nuevos datos que aporten un mayor número de evidencias.

La razón de este desfase puede ser la de que las "nuevas tecnologías" cambian muy rápidamente $y$, con ello, la posibilidad de detenerse y reflexionar supone la inmediata pérdida de actualidad. En algunos aspectos concretos probablemente sea así, pero creemos que, los aprendizajes de más calado y de más profundidad, mantienen su vigencia 
cuando son capaces de acompañar las distintas transformaciones tecnológicas mirando más allá de sus ventajas e inconvenientes.

Tampoco consideramos que éste sea un momento para, como profesionales, limitarnos a tomar partido a favor o en contra de este recurso a pesar de las muchas posibilidades y riesgos que entraña. Por el contrario, pensamos que, como ya se ha expuesto, es el momento para investigar y formarse, mientras acumulamos experiencia y observación que nos permita evaluar mejor lo que hacemos y cómo lo hacemos.

Cada persona, y por tanto cada paciente, tiene su particular estilo, su forma especial y única de "anudar el cuerpo, la palabra y la imagen" (en palabras de Ubieto, 2020), pero sin perder de vista que estamos tratando de una "representación" de la realidad; sorprendente e impactante, pero representación. Al fin y al cabo, como lo expresa Martínez Farrero, "[la pantalla] habla de mí, pero no soy yo, porque yo no puedo estar en dos sitios a la vez" (Martínez Farrero, 2020).

Lo que debería preocuparnos no es el recurso tecnológico, al que sin duda damos la bienvenida, sino el uso que hagamos de él. Si realizamos nuestro trabajo en un entorno social y temporal en el que el mercado global y el uso de las nuevas tecnologías marcan las directrices a seguir, es lógico pensar que estará influido por ellas, pero consideramos que es muy importante recordar una de las principales aportaciones de Richard Sennett (2000). Según este eminente sociólogo, el lema de este cambio globalizador y tecnológico es "nada a largo plazo", y este "es un principio que corroe la confianza, la lealtad y el compromiso mutuos" y añade: "para hacer frente a esta realidad actual, el desapego y la cooperación superficial son una armadura mejor que el comportamiento basado en los valores de lealtad y servicio".

Este sí que es nuestro verdadero reto como profesionales: hacer todo lo que esté a nuestro alcance para preservar la confianza y la lealtad en un compromiso que, para nosotros, sí que es a largo plazo.

\section{REFERENCIAS}

APA Joint Task Force for the Development of Telepsychology Guidelines for Psychologists. (2013). Guidelines for the practice of telepsychology. American Psychologist, 68(9), 791-800. http://dx.doi.org/10.1037/a0035001.

Cabré, V. \& Mercadal, J. (2016). Psicoterapia On-line. Escenario Virtual para una relación real en el espacio intermediario. Clínica e Investigación Relacional, Vol. 10 (2): 439-452. [ISSN 1988-2939] DOI: $10.21110 / 19882939.2016 .100206$ 
Drath, W. \& Necki, S. (2018). Online Psychotherapy: Polish psychotherapist perspective. Psychotherapia, Vol. 3 (186): 55-63.

Manfrida, G., Albertini, V. \& Eisenberg E. (2018). Psychotherapy and Technology: Relational Strategies and Techniques for Online Therapeutic Activity. In: Pereira R., Linares J. (eds) Clinical Interventions in Systemic Couple and Family Therapy. European Family Therapy Association Series. Springer, Cham. DOI: 10.1007/978-3-319-78521-9_9

Martínez Farrero, P. (2020). La "fatiga Zoom" i altres límits de la comunicació virtual. PsiAra Col-legi Oficial de Psicologia de Catalunya. Recuperado de: www.psiara.cat/print.asp?print=yes

Pieta, M.A. \& Gomes, W.B. (2014). Online therapy: viable or unviable? Psicologia : Ciencia e Profissao; Brasilia, Vol. 34(1): 18-31. DOI:10.1590/S1414-98932014000100003

Ribé, J. M. (2021). Técnica en psicoterapia analítica grupal. Experiencia desde la asistencia pública. Barcelona. Herder

Rollman, B.L., Herbeck, B. \& Abebe, K.Z. (2018) Effectiveness of Online Collaborative Care for Treating Mood and Anxiety Disorders in Primary Care: A Randomized Clinical Trial. JAMA Psychiatry. Vol.75(1):56-64. DOI:10.1001/jamapsychiatry.2017.3379

Schulze, N., Reuter, S.C., Kuchler, I., Reinke, B., Heinkelmann, L., Stöckigt, S., Siemoneit, H. \& Tonn, P. (2019). Differences in attitudes toward online interventions in psychiatry and psychotherapy between health care professionals and nenprofessionals: a survey. Telemedicidne and e-Health, Vol. 25(10). DOI: https://doi.org/10.108g/tmj.2018.0225

Sennett, R. (2000). La corrosión del carácter. Las consecuencias personales del trabajo en el nuevo capitalismo. Barcelona: Anagrama.

Stoll, J. \& Trachsek, M. (2019). Ethical Aspects of Online Psychotherapy. The Oxford Handbook of Psychotherapy Ethics. DOI: 10.1093/oxfordhb/9780198817338.013.64

Torre y Pardo (2018). Guía Para la Intervención Telepsicológica. Madrid: Colegio Oficial de Psicólogos de Madrid. Recuperado de: https://www.copmadrid.org/web/publicaciones/guia-para-laintervenciontelepsicologica

Ubieto, J.R. (2020). La 'fatiga Zoom', un nuevo cansancio. The conversation. Recuperado de: https://theconversation.com/la-fatiga-zoom-un-nuevo-cansancio-138913

Vaimberg, R. (2012). Bases de la psicoterapia e intervenciones online. Intercanvis, Vol. 28.

Zwerenz, R., Becker, J., Knickenberg, R.J., Siepmann, M.'Hagen, K. \& Beutel, M.E. (2017). Online Self-Help as an Add-On to Inpatient Psychotherapy: Efficacy of a New Blended Treatment Approach. Psychotherapy and Psychosomatics, Vol. 86: 341-350.

Original recibido con fecha: 12-9-2021 Revisado: 30-9-2021 Aceptado: 30/09/2021 